\title{
On the embedding of spacetime in five-dimensional Weyl spaces
}

\author{
${ }^{a}$ F. Dahia, ${ }^{b}$ G. A. T. Gomez, ${ }^{b}$ C. Romero \\ ${ }^{a}$ Departamento de Física, Universidade Federal de Campina Grande, \\ 58109-970, Campina Grande, Pb, Brazil \\ ${ }^{b}$ Departamento de Física, Universidade Federal da Paraíba, \\ C. Postal 5008, 58051-970 João Pessoa, Pb, Brazil \\ e-mail: cromero@fisica.ufpb.br, Brazil
}

November 2, 2018

\begin{abstract}
We revisit Weyl geometry in the context of recent higher-dimensional theories of spacetime. After introducing the Weyl theory in a modern geometrical language we present some results that represent extensions of Riemannian theorems. We consider the theory of local embeddings and submanifolds in the context of Weyl geometries and show how a Riemannian spacetime may be locally and isometrically embedded in a Weyl bulk. We discuss the problem of classical confinement and the stability of motion of particles and photons in the neighbourhood of branes for the case when the Weyl bulk has the geometry of a warped product space. We show how the confinement and stability properties of geodesics near the brane may be affected by the Weyl field. We construct a classical analogue of quantum confinement inspired in theoretical-field models by considering a Weyl scalar field which depends only on the extra coordinate.
\end{abstract}

\section{Introduction}

It has been suggested in the recent years that our ordinary spacetime may be viewed as a hypersurface embedded in a higher-dimensional manifold, often referred to as the bulk [1. As far as the geometry of this hypersurface is concerned, it has been generally assumed that it has a Riemannian geometrical structure. This assumption avoids possible conflicts with the well-established theory of general relativity which operates in a Riemannian geometrical frame. On the other hand, with very few exceptions, there has not been much discussion on what kind of geometry the bulk possesses, which is generally supposed to be also Riemannian. A very few attempts to broaden this scenario has appeared recently in the literature, where a non-Riemannian geometry, namely a Weyl 
geometry, is taken into consideration as a viable possibility to describe the bulk [2, 3, 4. However, a vast number of interesting non-Riemannian geometries are currently known and could be investigated in this context. It is presently our intention to carry out such a program of research, and a first step in this direction would be to consider the Weyl geometry [5], one of the simplest generalizations of Riemannian geometry.

The paper is organized as follows. We start in Section 2 with a modern definition of the Weyl geometry and state some results that represent straightforward extensions of Riemannian theorems. We proceed in Section 3 to consider the theory of local embeddings and submanifolds in the context of Weyl geometries. In Section 4 we show that a Riemannian spacetime may be embedded in a Weyl bulk and this constitutes one of our main results. Section 5 contains an application of the formalism to the problem of classical confinement and the stability of motion of particles and photons in the neighbourhood of branes. In Section 6 we show how the presence of a Weyl field may affect both the confinement and/or stablity of the particle's motion and discuss how a geometrical field, such as the Weyl field, may effectively act as a quantum scalar field, which in some theoretical-field modes is the responsible for the confinement of matter in the brane [6].

Section 7 we give a simple application of the ideas developed previously. We conclude, in Section 8, with some final remarks.

\section{Weyl geometry}

As is well known, the kind of geometrical structure conceived by H. Weyl in 1918, although admirably ingenious as an attempt to unify gravity with electromagnetism, turned out to be a failure as a physical theory. In fact, immediately after have been exposed to Weyl's ideas, Einstein raised strong objections to the adoption of Weyl geometry in the description of electromagnetic as well as gravitational phenomena [7]. Einstein's argument was that in a non-integrable geometry it would not be possible the existence of sharp spectral lines in the presence of an electromagnetic field since atomic clocks would depend on their past history [8]. It should be said, however, that a variant of Weyl geometries, known as Weyl integrable geometry, which does not suffer from the drawback pointed out by Einstein has attracted the attention of cosmologists some years ago 9 .

In this section we review some basic definitions and results that are valid in Riemannian and Weyl geometry. As we shall see, Weyl geometry may be viewed as a kind of generalization of Riemannian geometry, and some theorems that will be presented here are straightforward extensions of corresponding theorems of the former. However, these extensions have a different and new flavour especially when they are applied to study geodesic motion. Let us start with the definition of affine connection .

Definition. Let $M$ be a differentiable manifold and $T(M)$ the set of all differentiable vector fields on $M$. An affine connection is a mapping $\nabla: T(M) \times$ 
$T(M) \rightarrow T(M)$, which is denoted by $(U, V) \rightarrow \nabla_{U} V$, satisfying the following properties:

$$
\begin{aligned}
& \text { i) } \nabla_{f V+g U} W=f \nabla_{V} W+g \nabla_{U} W, \\
& \text { ii) } \nabla_{V}(U+W)=\nabla_{V} U+\nabla_{V} W, \\
& \text { iii) } \nabla_{V}(f U)=V[f] U+f \nabla_{V} U,
\end{aligned}
$$

where $V, U, W \in T(M), f$ and $g$ are $C^{\infty}$ scalar functions defined on $M$. An important result comes immediately from the above definition and allows one to define a covariant derivative along a differentiable curve.

Proposition. Let $M$ be a differentiable manifold endowed with an affine connection $\nabla, V$ a vector field defined along a differentiable curve $\alpha:(a, b) \subset$ $R \rightarrow M$. Then, there exists a unique rule which associates another vector field $\frac{D V}{d \lambda}$ along $\alpha$ to $V$, such that

$$
\begin{aligned}
& \text { i) } \frac{D(V+U)}{d \lambda}=\frac{D V}{d \lambda}+\frac{D U}{d \lambda}, \\
& \text { ii) } \frac{D(f V)}{d \lambda}=\frac{d f}{d \lambda} V+f \frac{D V}{d \lambda},
\end{aligned}
$$

where $\alpha=\alpha(\lambda)$ and $\lambda \in(a, b)$.

iii) If the vector field $U(\lambda)$ is induced by a vector field $\hat{U} \in T(M)$, then $\frac{D U}{d \lambda}=\nabla_{V} U$, where $V$ is the tangent vector to the curve $\alpha$, i.e $V=\frac{d}{d \lambda}$. For a proof of this proposition we refer the reader to [10. We now are ready for being introduced to the concept of parallel transport of a vector along a given curve.

Definition. Let $M$ be a differentiable manifold with an affine connection $\nabla, \alpha:(a, b) \in R \rightarrow M$ a differentiable curve on $M$, and $V$ a vector field defined along $\alpha=\alpha(\lambda)$. The vector field $V$ is said to be parallel if $\frac{D V}{d \lambda}=0$ for any value of the parameter $\lambda \in(a, b)$.

Among all admissible affine connections defined on a manifold, an important role in Riemannian and also in Weyl theory is played by a special class of connections, namely, the torsionless connections, as defined below.

Definition. We say that an affine connection $\nabla$ defined on $M$ is torsionless (or symmetric) if for any $U, V \in T(M)$ the following condition holds:

$$
\nabla_{V} U-\nabla_{U} V=[V, U]
$$

We now introduce the concept of Weyl manifold through the following definition.

Definition. Let $M$ be a differentiable manifold endowed with an affine connection $\nabla$, a metric tensor $g$ and a one-form field $\sigma$, called a Weyl field, globally defined in $M$. We say that $\nabla$ is Weyl-compatible ( or W-compatible) with $g$ if for any vector fields $U, V, W \in T(M)$, the following condition is satisfied:

$$
V[g(U, W)]=g\left(\nabla_{V} U, W\right)+g\left(U, \nabla_{V} W\right)+\sigma(V) g(U, W)
$$


This is, of course, a generalization of the idea of Riemannian compatibility between $\nabla$ and $g$. If the one-form $\sigma$ vanishes throughout $M$, we recover the Riemannian compatibility condition. It is therefore rather natural to expect that a generalized version of the Levi-Civita theorem holds if we restrict ourselves to torsionless connections. Indeed, we have the following result:

Theorem (Levi-Civita extended). In a given differentiable manifold $M$ endowed with a metric $g$ and a differentiable one-form field $\sigma$ defined on $M$, there exists only one affine connection $\nabla$ such that: $i) \nabla$ is torsionless; ii) $\nabla$ is W-compatible.

Proof. Let us first suppose that such $\nabla$ exists. Then, from (7) we have the following three equations

$$
\begin{aligned}
& V[g(U, W)]=g\left(\nabla_{V} U, W\right)+g\left(U, \nabla_{V} W\right)+\sigma(V) g(U, W) \\
& W[g(V, U)]=g\left(\nabla_{W} V, U\right)+g\left(V, \nabla_{W} U\right)+\sigma(W) g(V, U) \\
& U[g(W, V)]=g\left(\nabla_{U} W, V\right)+g\left(W, \nabla_{U} V\right)+\sigma(U) g(W, V)
\end{aligned}
$$

Adding (8) and (9) and subtracting (10), and also taking into account the torsionless condition (6), we are left with

$$
\begin{aligned}
g\left(\nabla_{W} V, U\right) & =\frac{1}{2} V[g(U, W)]+W[g(V, U)]-U[g(W, V)]-g([V, W], U) \\
& -g([W, U], V)-g([V, U], W)+\sigma(U) g(V, W)-\sigma(V) g(U, W)-\sigma(W) g(U, V)\}
\end{aligned}
$$

The above equation shows that the affine connection $\nabla$, if it exists, is uniquely determined from the metric $g$ and the Weyl field of one-forms $\sigma$. Now, to prove the existence of such a connection we just define $\nabla_{U} V$ by means of (11). At this point it is instructive to write (7) in a local coordinate system $\left\{x^{a}\right\}, a=1, \ldots, n$. Then, a straightforward calculation shows that one can express the components of the affine connection completely in terms of the components of $g$ and $\sigma$ :

$$
\Gamma_{b c}^{a}=\left\{\begin{array}{l}
a \\
b c
\end{array}\right\}-\frac{1}{2} g^{a d}\left[g_{d b} \sigma_{c}+g_{d c} \sigma_{b}-g_{b c} \sigma_{d}\right]
$$

where $\left\{\begin{array}{l}a \\ b c\end{array}\right\}=\frac{1}{2} g^{a d}\left[g_{d b, c}+g_{d c, b}-g_{b c, d}\right]$ denotes the Christoffel symbols of second kind.

At this stage let us note that the Weyl compatibility condition (7) may equivalently be interpreted as requiring that the covariant derivative of the metric tensor $g$ in the direction of a vector field $V \in T(M)$ do not vanish, as in Riemannian geometry, but, instead, that it be regulated by the Weyl field $\sigma$ defined in the manifold $M$. In other words, we must have

$$
\nabla g=\sigma \otimes g
$$

where $\sigma \otimes g$ denotes the direct product of $\sigma$ and $g$. It looks a bit surprising that this new requirement does not spoil the miraculous determinability of the connection $\nabla$ from $\sigma$ and $g$ only. [11]. A clear geometrical insight on the properties of Weyl parallel transport is given by the following proposition: 
Corollary Let $M$ be a differentiable manifold with an affine connection $\nabla$, a metric $g$ and a Weyl field of one-forms $\sigma$. If $\nabla$ is W-compatible, then for any smooth curve $\alpha=\alpha(\lambda)$ and any pair of two parallel vector fields $V$ and $U$ along $\alpha$, we have

$$
\frac{d}{d \lambda} g(V, U)=\sigma\left(\frac{d}{d \lambda}\right) g(V, U)
$$

where $\frac{d}{d \lambda}$ denotes the vector tangent to $\alpha$.

If we integrate the above equation along the curve $\alpha$, starting from a point $P_{0}=\alpha\left(\lambda_{0}\right)$ then we readily obtain

$$
g(V(\lambda), U(\lambda))=g\left(V\left(\lambda_{0}\right), U\left(\lambda_{0}\right)\right) e^{\int_{\lambda_{0}}^{\lambda} \sigma\left(\frac{d}{d \rho}\right) d \rho}
$$

Putting $U=V$ and denoting by $L(\lambda)$ the length of the vector $V(\lambda)$ at an arbitrary point $P=\alpha(\lambda)$ of the curve, then it is easy to see that in a local coordinate system $\left\{x^{a}\right\}$ the equation (14) reduces to

$$
\frac{d L}{d \lambda}=\frac{\sigma_{a}}{2} \frac{d x^{a}}{d \lambda} L
$$

Consider the set of all closed curves $\alpha:[a, b] \in R \rightarrow M$, i.e, with $\alpha(a)=\alpha(b)$. Then, the equation

$$
g(V(b), U(b))=g(V(a), U(a)) e^{\int_{a}^{b} \sigma\left(\frac{d}{d \lambda}\right) d \lambda}
$$

defines a holonomy group, whose elements are, in general, a composition of a homothetic transformation and an isometry. If we want the elements of this group to correspond to an isometry only, then we must have

$$
\oint \sigma\left(\frac{d}{d \lambda}\right) d \lambda=0
$$

for any loop. It follows, from Stokes' theorem, that $\sigma$ must be an exact form, that is, there exists a scalar function $\phi$, such that $\sigma=d \phi$. In this case we have what is often called in the literature a Weyl integrable manifold.

Weyl manifolds are completely caracterized by the triple $(M, g, \sigma)$, which we shall call a Weyl frame. It is interesting to see that the Weyl compatibility condition (14) remains unchanged when we go to another Weyl frame $(M, \bar{g}, \bar{\sigma})$ by performing the following simultaneous transformations in $g$ and $\sigma$ :

$$
\begin{gathered}
\bar{g}=e^{-\phi} g \\
\bar{\sigma}=\sigma-d \phi
\end{gathered}
$$

where $\phi$ is a scalar function defined on $M$. Clearly the conformal map (16) and the gauge transformation (17) define classes of equivalences in the set of Weyl frames. It is worth mentioning that the discovery that the compatibility requirement (14) is invariant under this group of transformations was what primarely led Weyl to his attempt at unifying gravity and electromagnetism, extending the concept of spacetime to that of a collection of manifolds equipped with a conformal structure, i.e, the spacetime would be viewed as a class $[g]$ of conformally equivalent Lorentzian metrics [5]. 


\section{Submanifolds and isometric embeddings in Weyl geometry}

Definition. Let $(M, g, \sigma)$ and $(\bar{M}, \bar{g}, \bar{\sigma})$ be differentiable Weyl manifolds of dimensions $m$ and $n=m+k$, respectively. A differentiable map $f: M \rightarrow \bar{M}$ is called an immersion if

i) the differential $f_{*}: T_{P}(M) \rightarrow T_{f(P)} \bar{M}$ is injective for any $P \in M$;

ii) $\sigma(V)=\bar{\sigma}\left(f_{*}(V)\right)$ for any $V \in T_{P}(M)$. The number $k$ is called the codimension of $f$. We say that the immersion $f: M \rightarrow \bar{M}$ is isometric at a point $P \in M$ if $g(U, V)=\bar{g}\left(f_{*}(U), f_{*}(V)\right)$ for every $U, V$ in the tangent space $T_{P}(M)$. If, in addition, $f$ is a homeomorphism onto $f(M)$, then we say that $f$ is an embedding. If $M \subset \bar{M}$ and the inclusion $i: M \subset \bar{M} \rightarrow \bar{M}$ is an embedding,

then $M$ is called a submanifold of $\bar{M}$.

It is important to note that locally any immersion is an embedding. Indeed, let $f: M \rightarrow \bar{M}$ be an immersion. Then, around each $P \in M$, there is a neighbourhood $U \subset M$ such that the restriction of $f$ to $U$ is an embedding onto $f(U)$. We may, therefore, identify $U$ with its image under $f$, so that locally we can regard $M$ as a submanifold embedded in $\bar{M}$, with $f$ actually being the inclusion map. Thus, we shall identify each vector $V \in T_{P}(M)$ with $f_{*}(V) \in$ $T_{f(P)}(\bar{M})$ and consider $T_{P}(M)$ as a subspace of $T_{f(P)}(\bar{M})$.

Now, in the vector space $T_{P}(\bar{M})$ the metric $\bar{g}$ allows one to make the decomposition $T_{P}(\bar{M})=T_{P}(M) \oplus T_{P}(M)^{\perp}$, where $T_{P}(M)^{\perp}$ is the orthogonal complement of $T_{P}(M) \subset T_{P}(\bar{M})$. That is, for any vector $\bar{V} \in T_{P}(\bar{M})$, with $P \in M$, we can decompose $\bar{V}$ into $\bar{V}=V+V^{\perp}, V \in T_{P}(M), V^{\perp} \in T_{P}(M)^{\perp}$.

Let us denote the Weyl connection on $\bar{M}$ by $\bar{\nabla}$. We now can prove the following proposition.

Proposition. If $V$ and $U$ are local vector fields on $M$, and $\bar{V}$ and $\bar{U}$ are local extensions of these fields to $\bar{M}$, then the Weyl connection $\nabla_{V} U$ will be given by

$$
\nabla_{V} U=(\bar{\nabla} \bar{V} \bar{U})^{\top}
$$

where $(\bar{\nabla} \bar{V} \bar{U})^{\top}$ is the tangential component of $\bar{\nabla} \bar{V} \bar{U}$.

Proof. We start with the equation which expresses the Weyl compatibility requirement

$$
\bar{V}[\bar{g}(\bar{U}, \bar{W})]=\bar{g}(\bar{\nabla} \bar{V} \bar{U}, \bar{W})+\bar{g}\left(\bar{U}, \bar{\nabla}_{\bar{V}} \bar{W}\right)+\bar{\sigma}(\bar{V}) \bar{g}(\bar{U}, \bar{W})
$$

where $\bar{V}, \bar{U}, \bar{W} \in T(\bar{M})$. Now, suppose that $\bar{V}, \bar{U}, \bar{W}$ are local extensions of the the vector fields $V, U, W$ to $\bar{M}$. Clearly, at a point $P \in M$, we have

$$
\bar{V}[\bar{g}(\bar{U}, \bar{W})]=V[\bar{g}(\bar{U}, \bar{W})]=V[g(U, W)]
$$

where we have taking into account that the inclusion of $M$ into $\bar{M}$ is isometric. On the other hand, evaluating separately each term of the right-hand side of (19) at $P$ yields

$$
\left.\bar{g}(\bar{\nabla} \bar{V} \bar{U}, \bar{W})=\bar{g}\left((\bar{\nabla} \bar{V} \bar{U})^{\top}+(\bar{\nabla} \bar{V} \bar{U})^{\perp}, \bar{W}\right)=\bar{g}\left((\bar{\nabla} \bar{V} \bar{U})^{\top}, \bar{W}\right)=g(\bar{\nabla} \bar{V} \bar{U})^{\top}, W\right)
$$


with an analogous expression for $\bar{g}(\bar{U}, \bar{\nabla} \bar{V} \bar{W})$. From the above equations and the fact that $\bar{\sigma}(\bar{V})=\sigma(V)$ we finally obtain

$$
V[g(U, W)]=g\left((\bar{\nabla} \bar{V} \bar{U})^{\top}, W\right)+g\left(U,(\bar{\nabla} \bar{V} \bar{W})^{\top}\right)+\sigma(V) g(U, W)
$$

From the Levi-Civita theorem extended to Weyl manifolds, which asserts the uniqueness of affine connection $\nabla$ in a Weyl manifold we conclude that (18) holds. In other words, the tangential component of the covariant derivative $\bar{\nabla} \bar{V} \bar{U}$, evaluated at points of $M$, is nothing more than the covariant derivative of the induced Weyl connection from the metric $g$ on $M$, defined by $g(V, U)=\bar{g}($ $\left.f_{*}(V), f_{*}(U)\right)$.

\section{Embedding the spacetime in a Weyl bulk}

Now that we know how the mechanism of embedding submanifolds in Weyl geometry works, we are led to ask the following question: Is it possible to have a Riemannian submanifold embedded in a Weyl ambient space? The answer to this question is given by the following argument. A Riemannian manifold is a particular case of a Weyl manifold, in which the Weyl field $\sigma$ vanishes. Therefore, a submanifold $M$ embedded in Weyl space $\bar{M}$ will be Riemannian if and only if the field of 1-forms $\sigma$ induced by pullback from $\bar{\sigma}$ vanishes throughout $M$. That is, the necessary and sufficient condition for $M$ to be an embedded Riemannian manifold is that $\sigma(V)=0$ for any $V \in T(M)$.

To illustrate the above, and having in view future applications, let us consider the case in which the manifold $\bar{M}$ is foliated by a family of submanifolds defined by $k$ equations $y^{A}=y_{o}^{A}=$ constant 1 1 , with the spacetime $M$ corresponding to one of these manifolds $y^{A}=y_{o}^{A}=$ constant. In local coordinates $\left\{y^{a}\right\}$ of $\bar{M}$ adapted to the embedding the condition $\sigma(V)=0$ reads $\sigma_{\alpha} V^{\alpha}=0$, where $\sigma=$ $\sigma_{a} d x^{a}$ and $V=V^{\beta} \partial_{\beta}$. In the case of a Weyl integrable manifold $\sigma=d \phi$. In this case $\sigma(V)=0$ for any $V \in T(M)$ if, and only if $\frac{\partial \phi}{\partial x^{\alpha}}=0$. Therefore, in a Weyl integrable manifold if the scalar field $\phi$ is a function of the extra coordinates only, then the spacetime submanifold $M$ embedded in the bulk $\bar{M}$ is Riemannian.

The fact that we may have a Riemannian spacetime $M$ embedded in a Weyl bulk $\bar{M}$ does not mean that physical or geometrical effects coming from the extra dimensions should be absent. A nice illustration of this point is given by the behaviour of geodesics near the $M$. In section 6 we shall examine how a Weyl field may affect the geodesic motion in the case of a bulk with a warped product geometry. We shall be interested particularly in the problem of confinement and stability of the motion of particles and photons near the spacetime submanifold. 12, 13

\footnotetext{
${ }^{1}$ From now on lower case Latin indices take value in the range $(0,1, \ldots,(n+3))$, while Greek indices run over $(0,1,2,3)$. The coordinates of a generic point $P$ of the manifold $\bar{M}$ will be denoted by $y^{a}=\left(x^{\alpha}, y^{4}, \ldots y^{n+3}\right)$, where $x^{\alpha}$ denotes the four-dimensional spacetime coordinates and $y^{A}(A>3)$ refers to the $n$ extra coordinates of $P$.
} 


\section{Geodesic motion in a Riemannian warped prod- uct space}

In this section let us consider the case where the geometry of the bulk contains two special ingredients: a) It is a Riemannian manifold and b) its metric has the structure of a warped product space 14. As is well known, the importance of warped product geometry is closely related to the so-called braneworld scenario [1. Let us start with the investigation of geodesics in warped product spaces, firstly considering the Riemannian case.

We define a warped product space in the following way. Let $(M, g)$ and $(N, h)$ be two Riemannian manifolds of dimension $m$ and $r$, with metrics $g$ and $h$, respectively. Suppose we are given a smooth function $f: N \rightarrow R$ (which will called warping function). Then we can construct a new Riemannian manifold by setting $\bar{M}=M \times N$ and defining a metric $\bar{g}=e^{2 f} g \oplus k$. Here, for simplicity, we shall take $M=M^{4}$ and $N=R$, where $M^{4}$ denotes a four-dimensional Lorentzian manifold with signature $(+---)$ (referred to as spacetime). In local coordinates $\left\{y^{a}=\left(x^{\alpha}, y^{4}\right\}\right.$ the line element corresponding to this metric will be written as 2

$$
d S^{2}=\bar{g}_{a b} d y^{a} d y^{b}
$$

The equations of geodesics in the five-dimensional space $\bar{M}$ will be given by

$$
\frac{d^{2} y^{a}}{d \lambda^{2}}+{ }^{(5)} \Gamma_{b c}^{a} \frac{d y^{b}}{d \lambda} \frac{d y^{c}}{d \lambda}=0,
$$

where $\lambda$ is an affine parameter and ${ }^{(5)} \Gamma_{b c}^{a}$ denotes the 5D Christoffel symbols of the second kind defined by ${ }^{(5)} \Gamma_{b c}^{a}=\frac{1}{2} \bar{g}^{a d}\left(\bar{g}_{d b, c}+\bar{g}_{d c, b}-\bar{g}_{b c, d}\right)$. Denoting the fifth coordinate $y^{4}$ by $y$ and the remaining coordinates $y^{\mu}$ (the "spacetime" coordinates) by $x^{\mu}$, i.e. $y^{a}=\left(x^{\mu}, y\right)$, we can easily show that the " $4 \mathrm{D}$ part" of the geodesic equations (22) can be rewritten in the form

$$
\frac{d^{2} x^{\mu}}{d \lambda^{2}}+{ }^{(4)} \Gamma_{\alpha \beta}^{\mu} \frac{d x^{\alpha}}{d \lambda} \frac{d x^{\beta}}{d \lambda}=\xi^{\mu},
$$

where

$$
\begin{aligned}
\xi^{\mu} & =-{ }^{(5)} \Gamma_{44}^{\mu}\left(\frac{d y}{d \lambda}\right)^{2}-2^{(5)} \Gamma_{\alpha 4}^{\mu} \frac{d x^{\alpha}}{d \lambda} \frac{d y}{d \lambda} \\
& -\frac{1}{2} \bar{g}^{\mu 4}\left(\bar{g}_{4 \alpha, \beta}+\bar{g}_{4 \beta, \alpha}-\bar{g}_{\alpha \beta, 4}\right) \frac{d x^{\alpha}}{d \lambda} \frac{d x^{\beta}}{d \lambda},
\end{aligned}
$$

and ${ }^{(4)} \Gamma_{\alpha \beta}^{\mu}=\frac{1}{2} \bar{g}^{\mu \nu}\left(\bar{g}_{\nu \alpha, \beta}+\bar{g}_{\nu \beta, \alpha}-\bar{g}_{\alpha \beta, \nu}\right)$

At this point we turn our attention to the five-dimensional brane-world scenario, where the bulk corresponds to the five-dimensional manifold $\bar{M}$, which, as in the previous section, is assumed to be foliated by a family of submanifolds (in this case, hypersurfaces) defined by the equation $y=$ constant.

\footnotetext{
${ }^{2}$ Throughout this section Latin indices take values in the range $(0,1, \ldots 4)$ while Greek indices run from $(0,1,2,3)$.
} 
It turns out that the geometry of a generic hypersurface, say $y=y_{0}$, will be determined by the induced metric $g_{\alpha \beta}(x)=\bar{g}_{\alpha \beta}\left(x, y_{0}\right)$. Thus, on the hypersurface we have

$$
d s^{2}=\bar{g}_{\alpha \beta}\left(x, y_{0}\right) d x^{\alpha} d x^{\beta} .
$$

We see then that the quantities ${ }^{(4)} \Gamma_{\alpha \beta}^{\mu}$ which appear on the left-hand side of Eq. (23) are to be identified with the Christoffel symbols associated with the induced metric in the leaves of the foliation defined above.

Let us now consider the class of warped geometries given by the following line element

$$
d S^{2}=e^{2 f} g_{\alpha \beta} d x^{\alpha} d x^{\beta}-d y^{2},
$$

where $f=f(y)$ and $g_{\alpha \beta}=g_{\alpha \beta}(x)$. For this metric it is easy to $\operatorname{se}^{3}$ that ${ }^{(5)} \Gamma_{44}^{\mu}=0$ and ${ }^{(5)} \Gamma_{4 \nu}^{\mu}=\frac{1}{2} \bar{g}^{\mu \beta} \bar{g}_{\beta \nu, 4}=f^{\prime} \delta_{\nu}^{\mu}$, where a prime denotes a derivative with respect to $y$. Thus in the case of the warped product space (25) the righthand side of Eq. (23) reduces to $\xi^{\mu}=-2 f^{\prime} \frac{d x^{\mu}}{d \lambda} \frac{d y}{d \lambda}$ and the $4 \mathrm{D}$ part of the geodesic equations becomes

$$
\frac{d^{2} x^{\mu}}{d \lambda^{2}}+{ }^{(4)} \Gamma_{\alpha \beta}^{\mu} \frac{d x^{\alpha}}{d \lambda} \frac{d x^{\beta}}{d \lambda}=-2 f^{\prime} \frac{d x^{\mu}}{d \lambda} \frac{d y}{d \lambda} .
$$

On the other hand the geodesic equation for the fifth coordinate $y$ in the warped product space becomes

$$
\frac{d^{2} y}{d \lambda^{2}}+f^{\prime} e^{2 f} g_{\alpha \beta} \frac{d x^{\alpha}}{d \lambda} \frac{d x^{\beta}}{d \lambda}=0 .
$$

By restricting ourselves to $5 \mathrm{D}$ timelike geodesics $\left(\bar{g}_{a b} \frac{d y^{a}}{d \lambda} \frac{d y^{b}}{d \lambda}=1\right)$ we can readily decouple the above equation from the $4 \mathrm{D}$ spacetime coordinates to obtain

$$
\frac{d^{2} y}{d \lambda^{2}}+f^{\prime}\left(1+\left(\frac{d y}{d \lambda}\right)^{2}\right)=0 .
$$

Similarly, to study the motion of photons in 5D, we must consider the null geodesics $\left(\bar{g}_{a b} \frac{d y^{a}}{d \lambda} \frac{d y^{b}}{d \lambda}=0\right)$, in which case Eq. (27) becomes

$$
\frac{d^{2} y}{d \lambda^{2}}+f^{\prime}\left(\frac{d y}{d \lambda}\right)^{2}=0
$$

Equations (28) and (29) are ordinary differential equations of second-order which, in principle, can be solved if the function $f^{\prime}=f^{\prime}(y)$ is known. A qualitative picture of the motion in the fifth dimension may be obtained without

\footnotetext{
${ }^{3}$ In the above calculation we have used the fact that the matrix $g_{\alpha \beta}$ has an inverse $g^{\alpha \beta}$, that is, $g^{\mu \beta} g_{\beta \nu}=\delta_{\nu}^{\mu}$. This may be easily seen since by definition $\operatorname{det} g=-\operatorname{det} \bar{g} \neq 0$.
} 
the need to solve (28) and (29) analytically [13]. This is done by defining the variable $q=\frac{d y}{d \lambda}$ and then investigating the autonomous dynamical system [15]

$$
\begin{aligned}
& \frac{d y}{d \lambda}=q \\
& \frac{d q}{d \lambda}=P(q, y)
\end{aligned}
$$

with $P(q, y)=-f^{\prime}\left(\epsilon+q^{2}\right)$, where $\epsilon=1$ in the case of (28) (corresponding to the motion of particles with nonzero rest mass) and $\epsilon=0$ in the case of (29) (corresponding to the motion of photons). In the investigation of dynamical systems a crucial role is played by their equilibrium points, which in the case of system (30) are given by $\frac{d y}{d \lambda}=0$ and $\frac{d q}{d \lambda}=0$. The knowledge of these points together with their stability properties provides a great deal of information on the types of behaviour allowed by the system.

\subsection{The case of massive particles}

In the case of nonzero rest mass particles, the motion in the fifth dimension is governed by the dynamical system

$$
\begin{aligned}
& \frac{d y}{d \lambda}=q \\
& \frac{d q}{d \lambda}=-f^{\prime}\left(1+q^{2}\right)
\end{aligned}
$$

The critical points of (31) are given by $q=0$ and the zeros of the function $f^{\prime}(y)$ (if they exist) which we generically denote by $y_{0}$. These solutions, pictured as isolated points in the phase plane, correspond to curves which lie entirely on a hypersurface $M$ of our foliation (since for them $y=$ constant). It turns out that these curves are timelike geodesics with respect to the hypersurface induced geometry [13].

To obtain information about the possible modes of behaviour of particles and light rays in such hypersurfaces, it is important to study the nature and stability of the corresponding equilibrium points. This can be done by linearising equations (31) and studying the eigenvalues of the corresponding Jacobian matrix about the equilibrium points. Assuming that the function $f^{\prime}(y)$ vanishes, at least at one point $y_{0}$, it can readily be shown that the corresponding eigenvalues are determined by the sign of the second derivative $f^{\prime \prime}\left(y_{0}\right)$, at the equilibrium point, and some possibilities arise for the equilibrium points of the dynamical system (31) [13. We shall discuss only the three following cases.

Case I. If $f^{\prime \prime}\left(y_{0}\right)>0$, then the equilibrium point $\left(q=0, y=y_{0}\right)$ is a center. This corresponds to the case in which the massive particles oscillate about the hypersurface $M\left(y=y_{0}\right)$. Such cyclic motions are independent of the ordinary $4 \mathrm{D}$ spacetime dimensions, and, except for the conditions $f^{\prime}\left(y_{0}\right)=0$ and $f^{\prime \prime}\left(y_{0}\right)>0$, the warping function $f(y)$ remains completely arbitrary.

Case II. If $f^{\prime \prime}\left(y_{0}\right)<0$, then the point $\left(q=0, y=y_{0}\right)$ is a saddle point. In this case the solution corresponding to the equilibrium point is highly unstable 
and the smallest transversal perturbation in the motion of particles along the brane will cause them to be expelled into the extra dimension. An example of this highly unstable "confinement" at the hypersurface $y=0$ is provided by Gremm's warping function [16]

$$
f(y)=-b \ln \cosh (c y)
$$

where $b$ and $c$ are positive constants.

Case III. There are no equilibrium points at all. The warping function $f(y)$ does not have any turning points for any value of $y$. This implies that in this case we cannot have confinement of classical particles to hypersurfaces solely due to gravitational effects. An example of this situation is illustrated by the warping function $f(y)=\frac{1}{2} \ln \left(\Lambda y^{2} / 3\right)$ considered in [17]. In similar fashion, note that for large values of $y$ the warping function (33) approaches that of the Randall-Sundrum metric [18])

$$
d s^{2}=e^{-2 k|y|} \eta_{\alpha \beta} d x^{\alpha} d x^{\beta}-d y^{2},
$$

where $k$ is a constant. In this case $f^{\prime}(y)=\mp k$ according to whether $y$ is positive or negative. Again, there exist no equilibrium points, and therefore no confinement of particles is possible due only to gravity.

\subsection{The case of photons}

The motion of photons is governed by the dynamical system

$$
\begin{aligned}
& \frac{d y}{d \lambda}=q \\
& \frac{d q}{d \lambda}=-f^{\prime} q^{2}
\end{aligned}
$$

The equilibrium points in this case are given by $q=0$, so they consist of a line of equilibrium points along the $y$-axis, with eigenvalues both equal to zero. Any point along the $y$-axis is an equilibrium point and corresponds to a $5 \mathrm{D}$ null geodesics in the hypersurface $y=$ constant. The existence of photons confined to hypersurfaces does not depend upon the warping factor [13.

As is well known, in the brane-world scenario the stability of the confinement of matter fields at the quantum level is made possible by assuming an interaction of matter with a scalar field. An example of how this mechanism works is clearly illustrated by a field-theoretical model devised by Rubakov, in which fermions may be trapped to a brane by interacting with a scalar field that depends only on the extra dimension [6]. On the other hand, the kind of confinement we are concerned with is purely geometrical, and that means the only force acting on the particles is the gravitational force. In a purely classical (non-quantum) picture, one would like to have effective mechanisms other than a quantum scalar field in order to constrain massive particles to move on hypersurfaces in a stable way. At this point at least two possibilities come to our mind. One is to assume a direct interaction between the particles and a physical scalar field. 
Following this approach it has been shown that stable confinement in a thick brane is possible by means of a direct interaction of the particles with a scalar field through a modification of the Lagrangian of the particle [19]. Another approach would appeal to pure geometry: for instance, modelling the bulk with a Weyl geometrical structure. As we shall see, in this case the Weyl field may provide the mechanism necessary for confinement and stabilization of the motion of particles in the brane.

\section{Geodesic motion in the presence of a Weyl field}

The question we want to discuss now is: What happens with the geodesic motion pictured in the previous section when we "turn on" a Weyl field? For simplicity, let us consider the case when the warped product bulk is an integrable Weyl manifold $(\bar{M}, \bar{g}, \phi)$. As we have seen in Section IV, if the Weyl scalar depends only on the extracoordinates, then the Weyl field of 1-forms $\sigma=d \phi$ induced on the hypersurfaces of the foliation defined above vanishes. Indeed, any tangent vector $V$ of a given leaf $M$ has the form $V=V^{\alpha} \partial_{\alpha}$. Thus, we have $\sigma(V)=$ $d \phi(V)=V^{\alpha} \frac{\partial \phi}{\partial x^{\alpha}}=0$. Therefore, if $M$ represents our spacetime embedded in a integrable Weyl bulk $\bar{M}$ with $\phi=\phi(y)$, then we can be sure that $M$ has a Riemannian structure.

We have seen in Section II, that in a Weyl manifold the coefficients of the Weyl connection $\Gamma_{b c}^{a}$ are related to the Christoffel symbols through the equation

$$
\Gamma_{b c}^{a}=\left\{\begin{array}{c}
a \\
b c
\end{array}\right\}-\frac{1}{2} g^{a d}\left[g_{d b} \sigma_{c}+g_{d c} \sigma_{b}-g_{b c} \sigma_{d}\right]
$$

From (12) it is not difficult to show that the geodesic equation for the fifth coordinate $y$ in this warped product space leads, for massive particles, to the equation

$$
\frac{d^{2} y}{d \lambda^{2}}+f^{\prime}\left(1+\left(\frac{d y}{d \lambda}\right)^{2}\right)-\phi^{\prime}\left(\frac{1}{2}+\left(\frac{d y}{d \lambda}\right)^{2}\right)=0 .
$$

where $\phi^{\prime}=\frac{d \phi}{d y}$.

On the other hand, for photons we now have

$$
\frac{d^{2} y}{d \lambda^{2}}+\left(f^{\prime}-\phi^{\prime}\right)\left(\frac{d y}{d \lambda}\right)^{2}=0 .
$$

The equations (36) and (37) respectively define the following dynamical systems:

$$
\begin{aligned}
& \frac{d y}{d \lambda}=q \\
& \frac{d q}{d \lambda}=\left(\phi^{\prime}-f^{\prime}\right) q^{2}+\frac{\phi^{\prime}}{2}-f^{\prime}
\end{aligned}
$$




$$
\begin{aligned}
& \frac{d y}{d \lambda}=q \\
& \frac{d q}{d \lambda}=\left(\phi^{\prime}-f^{\prime}\right) q^{2}
\end{aligned}
$$

Clearly, the presence of the derivative of the Weyl scalar in the above equations may completely change the picture of the solutions determined by the dynamical system considered in the previous section. This is because the existence of equilibrium points, their topology and stability properties now depends not only on the values the derivatives the warping function take at the brane, but also on the derivatives of the Weyl scalar field $\phi(y)$.

Finally, note that in the case of photons the Weyl scalar field $\phi$ has no influence on the confinement. This can be easily explained by the fact that, according to (??) and (17), the presence of a scalar Weyl is equivalent to perform a conformal transformation in the Riemannian metric $\bar{g}=e^{2 f} g \oplus k$. This essentially results in changing the warping function from $f$ to $f-\phi / 2$. Because the existence of confined photons in the hypersurface is independent of the warping function [13, the Weyl scalar has no effect in the confinement. This interesting property can also be explained by the fact that a conformal transformation does not alter the light-cone structure of a manifold.

\section{$7 \quad$ A simple example}

As an illustration of the results obtained in the previous section, let us consider the five-dimensional Riemannian space $\bar{M}$ endowed with a Mashhoon-Wessontype metric [17]

$$
d S^{2}=\frac{\Lambda^{2}}{3} y^{2} g_{\alpha \beta} d x^{\alpha} d x^{\beta}-d y^{2} .
$$

As we have remarked in Section 5, in this case there is no confinement of particles in the hypersurfaces $y=$ const. Now let us "turn on" a Weyl field in the space $\bar{M}$ by chosing, for instance,

$$
\phi=\ln y^{2}+K\left(y-y_{0}\right)^{2}
$$

where $K$ is a constant. It is not difficult to verify that the Weyl scalar field will act as a confining field, trapping massive particles in the hypersurface $y=$ $y_{0}$. A simple calculation shows that if $K>0$ we are in the presence of a kind of confinement where particles lying near the hypersurface $y=y_{0}$ will oscillate about it, entering and leaving the hypersurface indefinitely (see ([13]), for details). On the other hand, if $K<0$, the classical confinement is highly unstable. Clearly, the same procedure can also be used to stabilize the motion of the trapped particles in the case of Gremm's warping function (33). Finally, note that since $\phi$ depends only on the extra coordinate $y$, the Riemannian character of the hypersurfaces $y=$ const is not affected by the presence of the Weyl field. 


\section{Final Remarks}

An important class of higher-dimensional models in the braneworld scenario share the following three properties: a) our spacetime is viewed as four-dimensional Riemannian hypersurface (brane) embedded in a five-dimensional Riemannian manifold (bulk); b) the geometry of the bulk space is characterized by a warped product space; c) fermionic matter is confined to the brane by means of an interaction of the fermions with a scalar field which depends only on the extra dimension. In this article we have considered the possibility of describing the bulk by a non-Riemannian geometry, namely, a Weyl manifold. For a class of Weyl fields, the geometry induced on the brane has a Riemannian structure. However, the confinement and stability properties of geodesics near the brane may be affected by the Weyl field. Taking this fact into account we have constructed a classical analogue of the quantum confinement by considering a Weyl scalar field which also depends only on the extra coordinate. In a certain way, this Weyl scalar field, which has a purely geometrical nature, seems to mimic the quantum scalar field that is responsible for the confinement in field-theoretical models [6].

Throughout this article, we have assumed the existence a priori of a Weyl field and have not discussed the dynamics of this field and how it would determine the geometry of the bulk. We leave this subject for a future work.

Finally, as far as the geometrical structure of Weyl inspired higher-dimensional model is concerned, one would like to look at the embedding properties of the bulk space. We now know that embedding theorems of differential geometry are of vital importance for some higher-dimensional theories of spacetime. This is particularly true in the case of the induced-matter proposal [20. Thus, an interesting question is how to formulate the analogous of the Campbell-Magaard theorem and its extended versions in the context of a Weyl geometry [21]. An answer to this question would, in principle, tell us what kind of Weyl bulk space is admissible if matter and fields are to be generated from the extra dimensions, pretty much in the same way as in the case of the (Riemannian) induced matter proposal and Kaluza-Klein theories.

\section{Acknowledgement}

The authors would like to thank CNPq-FAPESQ (PRONEX) for financial support.

\section{References}

[1] See, for instance, R. Maartens, Brane-World Gravity, Living Rev. Relativity 7 (2004). 
[2] M. Israelit, Found. Phys. 35, 1725 (2005).

[3] O. Arias, R. Cardenas, I. Quiros, Nucl.Phys. B 643, 187 (2002).

[4] N. Barbosa-Cendejas and A. Herrera-Aguilar, Phys. Rev. D 73, 084022 (2006).

[5] H. Weyl, Sitzungesber Deutsch. Akad. Wiss. Berlin, 465 (1918). H. Weyl, Space, Time, Matter (Dover, New York, 1952)

[6] V. A. Rubakov, Phys. Usp. 44, 871 (2001). e-Print: hep-ph/0104152.

[7] A. Pais, Subtle is the Lord (Oxford University Press, 1983)

[8] A nice account of Weyl's ideas as well as their refutation may be found in W. Pauli, Theory of Relativity (Dover, New York, 1981). See, also, L. O'Raiefeartaigh and N. Straumann, Rev. Mod. Phys. 72, 1 (2000).

[9] M. Novello, Theoretical Cosmology in Proceedings of the Seventh Brazilian School of Cosmology and Gravitation, Ed. M. Novello (Editions Frontières, Rio de Janeiro, 1994). M. Novello, L. A. R. Oliveira, J. M. Salim, E. Elbaz, Int. J. Mod. Phys. D 1, 641 (1993). J. M. Salim and S. L. Sautú, Class. Quantum Grav. 13, 353 (1996).

[10] See for instance, M. P. do Carmo, Riemannian Geometry ( Birkhauser, Boston, 1991)

[11] Berger refers to the property of an affine connection being completely determined from the metric in Riemannian geometry as a "miracle". See M. Berger, A Panoramic View of Riemannian Geometry (Springer, Berlin, 2007).

[12] S. S. Seahra, Phys. Rev. D 68:104027(2003). e-Print: hep-th/0309081

[13] F. Dahia, L. F. P. da Silva, C. Romero, R. Tavakol, J. Math. Phys. 48, 72501 (2007).

[14] G. N. Felder, A. Frolov and L. Kofman, Class. Quantum Grav. 19, 2983 (2002); M. Arik, A. Baykal, M. C. Çalik, D. Çiftçi, and Ö. Delice, Phys. Rev. D 68, 123503 (2003). R. L. Bishop and B. O. O'Neill, Trans. Am. Math. Soc. 145, 1 (1969); J. K. Beem and P. E. Ehrlich, Math. Proc. Camb. Phil. Soc. 85, 161 (1979); J. Carot and J. da Costa, Class. Quantum Grav. 10, 461 (1993).

[15] See, for instance, A. A. Andronov, A. E. Leontovich, I. I. Gordon, A. G. Maier, Qualitative Theory of Second Order Dynamical Systems (Wiley, New York, 1973).

[16] M. Gremm, Phys. Lett. B 478, 434 (2000). 
[17] P. S. Wesson, B. Mashhoon and H. Liu, Mod. Phys. Lett. A 12, 2309 (1997). B. Mashhoon and P. S. Wesson, Class. Quantum Grav. 21, 3611 (2004).

[18] L. Randall and R. Sundrum, Phys. Rev. Lett. 83, 3370 (1999); L. Randall and R. Sundrum, Phys. Rev. Lett. 83, 4690 (1999).

[19] F. Dahia and C. Romero, Phys. Lett. B 651, 232 (2007).

[20] P. S. Wesson, Space-Time-Matter (World Scientific, 1999). J. M. Overduin and P. S. Wesson, Phys. Rep. 283, 302 (1997). P. S. Wesson, FiveDimensional Physics (World Scientific, 2006).

[21] C. Romero, R. Tavakol, and R. Zalaletdinov, Gen. Rel. Grav. 28, 365 (1995). F. Dahia and C. Romero, J. Math. Phys. 43, 5804 (2002). E. Anderson and J. E. Lidsey, Class. Quant. Grav.18, 4831 (2001). F. Dahia and C. Romero, J. Math. Phys. 43, 3097 (2002). E. Anderson, F. Dahia, James E. Lidsey, C. Romero, J. Math.Phys. 44, 5108 (2003). F. Dahia and C. Romero, Class. Quant. Grav. 21, 927 (2004). F. Dahia and C. Romero, Class. Quant. Grav. 22, 5005 (2005).

[22] T. Kaluza, Sitz. Preuss. Akad. Wiss. 33, 966 (1921). O. Klein, Z. Phys. 37, 895 (1926). T. Appelquist, A. Chodos and P. Freund, Modern Kaluza-Klein Theories (Addison-Wesley, Menlo Park, 1987) 